\title{
TORSIONAL RIGIDITY, PRINCIPAL FREQUENCY, ELECTROSTATIC CAPACITY AND SYMMETRIZATION*
}

\author{
BY \\ GEORGE PÓLYA \\ Stanford University
}

Symmetrization is a geometric operation invented by the Swiss mathematician Jacob Steiner. It was observed recently by Mr. Szegö and the author ${ }^{1}$ that this geometric operation has definite simple connections with important physical quantities, especially with those mentioned in the title of this paper. This observation leads to many curious consequences some of which may have practical importance.

When the present author was about to present the principal properties of symmetrization in the seminar of Professor Timoshenko, one of the latter's remarks suggested a new version of the method given in the aforementioned paper. This new version will be presented here, as intuitively as possible, and with a numerical illustration, see Sec. 6 (I). As another application, the first proof of an important conjecture of Saint Venant will be given in Sec. 6 (III). For further consequences and several allied results, the reader should refer to the joint papers of Mr. Szegö and the author. ${ }^{1,2}$

1. Definition of symmetrization. Symmetrization applies to plane and solid figures.

Symmetrization in a plane is performed with respect to a specified straight line, called the line of symmetrization. For example, symmetrization with respect to the line

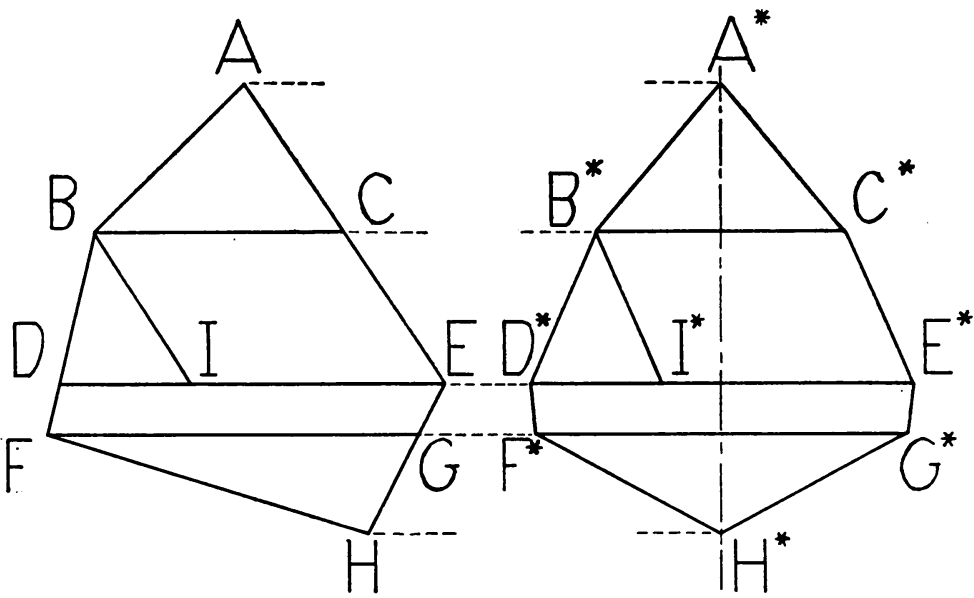

FIG. 1

${ }^{*}$ Received Dec. 20, 1947. The investigation here presented was carried out within the framework of an Office of Naval Research project directed by Mr. G. Szegö and the author.

${ }^{1}$ G. Polya and G. Szegö, Inequalities for the capacity of a condenser, Amer. J. Math., 67, 1-32 (1945). See also G. Szegö, On the capacity of a condenser, Bulletin Amer. Math. Soc. 51, 325-350 (1945) and G. Pólya, Sur la fréquence fondamentale des membranes vibrantes et la résistance élastique des tiges d la torsion, C. R. Ac. Sci. Paris, 225, 346-348 (1947).

${ }^{2}$ G. P6́lya and G. Szegö, Approximations and bounds for the electrostatic capacity and for similar physical quantities. In preparation. 
$A^{*} H^{*}$ of Fig. 1 transforms the pentagon $A B F H E$ into the octagon $A^{*} B^{*} D^{*} F^{*} H^{*} G^{*} E^{*} C^{*}$. The original figure is conceived as consisting of line-segments parallel to each other and perpendicular to the line of symmetrization. Each segment is shifted along its own line into a position where it is bisected by the line of symmetrization. The segments in their new positions form the new, transformed, symmetrized figure. Figure 4 shows the effect of two successive symmetrizations. The unsymmetric quadrilateral is symmetrized with respect to the horizontal line I into another quadrilateral (which has, of course, one axis of symmetry). The latter, symmetrized with respect to the vertical line II, is changed into a rhombus. Also, Fig. 3 shows two successive symmetrizations. The rectangle marked with $\Lambda^{\prime}$, symmetrized with respect to the line $I$, is transformed into a rhombus, and this, again, symmetrized with respect to the line II, into another rectangle marked with $\Lambda^{\prime \prime}$.

Symmetrization in space is performed with respect to a specified plane, called "the plane of symmetrization". The original solid is conceived as consisting of parallel linesegments perpendicular to the plane of symmetrization. These segments are shifted, each along its own line, to a position where they are bisected by the plane of symmetrization and form, in their new positions, the transformed solid. For example, in Fig. 2, the plane of symmetrization is perpendicular to the horizontal paper of the drawing which it cuts along $A^{*} C^{*}$. In this Fig. 2, on the left, closed contour lines are

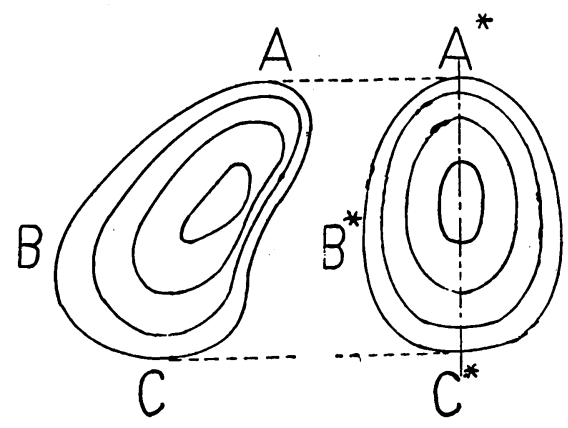

Frg. 2

shown, representing a hill in the terrain. This is transformed by symmetrization with respect to the vertical plane through $A^{*} C^{*}$ into a symmetrical hill. ${ }^{3}$

2. Influence of symmetrization on some geometrical quantities. Jacob Steiner found some interesting properties of symmetrization of which we must make essential use later. We summarize these properties in four statements of which the first two are concerned with symmetrization in a plane and the last two with symmetrization in space.

I. Symmetrization leaves the area unchanged.

II. Symmetrization decreases the perimeter.

III. Symmetrization leaves the volume unchanged.

IV. Symmetrization decreases the surface-area.

Statements I, II and III are almost immediate. We give a few hints about the elementary geometric considerations which constitute their proof.

${ }^{3}$ In the foregoing explanation we restricted ourselves to figures the boundary of which is not cut in more than two points by any perpendicular to the line, or plane, of symmetrization. It is enough for an introductory treatment to consider this simplest case. 
In dealing with symmetrization in a plane, we may restrict our considerations to polygons. In fact, a curved line can be regarded as the limiting case of a polygon. Let us divide the polygon which we intend to symmetrize by parallels passing through its vertices and perpendicular to the line of symmetrization. The polygon will be divided into a few triangles ( $\triangle A B C$ and $\triangle F G H$ in Fig. 1) and several trapezoids ( $B C E D, D E G F)$. Such a trapezoid is changed into another trapezoid by symmetrization $(B C E D$ into $\left.B^{*} C^{*} E^{*} D^{*}\right)$. These two trapezoids, however, the original and the symmetrized, have the same lower base, the same upper base, the same altitude, and, therefore, the same area. That a triangle is also symmetrized into another with the same area (as $\triangle A B C$ into $\triangle A^{*} B^{*} C^{*}$ ) is still easier to see. And so assertion $\mathrm{I}$ is proved.

In discussing II, we again consider polygons, divided by parallels as before. We must know the elementary proposition that, of all triangles having the same base and the same area the isosceles has the shortest perimeter. ${ }^{4}$ For example, in Fig. 1, the broken line $B^{*} A^{*} C^{*}$, belonging to an isosceles triangle, is shorter than the corresponding broken line $B A C$ in the non-symmetrized figure. (Consider the line $A A^{*}$ as a mirror; then $B^{*} A^{*} C^{*}$ is a possible path of light, but not $B A C$ which does not include equal angles with the mirror-and light chooses the shortest path.) We can also compare the sum of the two slanting sides of a trapezoid before and after symmetrization; this sum is shorter after. (For example, $D^{*} B^{*}+C^{*} E^{*}$, equal to the broken line $D^{*} B^{*} I^{*}$ belonging to the isosceles $\triangle D^{*} B^{*} I^{*}$, is shorter than $D B+C E$, equal to $D B I$.) Summing up such inequalities over all trapezoids and triangles into which the polygons, before and after symmetrization, are divided, we can justify II.

We do not give a formal proof for III. We just observe that a solid, undergoing symmetrization, can be conceived as consisting of elongated, slender prisms (matches of unequal lengths) all perpendicular to the plane of symmetrization. In the process of symmetrization these prisms (matches) are shifted, each in the line of its altitude, till they are bisected by the plane of symmetrization. Yet such shifting does not alter the volume of the prisms. This remark renders III evident.

The proof of assertion IV by elementary geometry is more difficult. If the reader does not wish to take IV for granted on the basis of the obvious analogy of I and II with III and IV, he may read the following proof which makes use of coordinates and calculus.

We introduce rectangular coordinates. We take the $y, z$-plane as the plane of symmetrization. (And we take the $x, y$-plane as the horizontal plane of the drawing. Thus, in Fig. 2, the line $A^{*} C^{*}$ represents the $y$-axis.) A straight line parallel to the $x$-axis that cuts the surface of the given solid, intersects it at two points which we call $\left(x_{1}, y, z\right)$ and $\left(x_{2}, y, z\right)$. We choose the notation so that $x_{1}<x_{2}$. The area of the surface is expressed by

$$
\iint\left[1+\left(\frac{\partial x_{1}}{\partial y}\right)^{2}+\left(\frac{\partial x_{1}}{\partial z}\right)^{2}\right]^{1 / 2} d y d z+\iint\left[1+\left(\frac{\partial x_{2}}{\partial y}\right)^{2}+\left(\frac{\partial x_{2}}{\partial z}\right)^{2}\right]^{1 / 2} d y d z
$$

We introduce the abbreviations

$$
\frac{\partial x_{1}}{\partial y}=Y_{1}, \quad \frac{\partial x_{1}}{\partial z}=Z_{1}, \quad \frac{\partial x_{2}}{\partial y}=Y_{2}, \quad \frac{\partial x_{2}}{\partial z}=Z_{2} .
$$

${ }^{4}$ See e.g. R. Courant and H. Robbins, What is mathematics?, pp. 330-333. 
Then we can express the area of the surface in the form

$$
\iint\left\{\left[1+Y_{1}^{2}+Z_{1}^{2}\right]^{1 / 2}+\left[1+Y_{2}^{2}+Z_{2}^{2}\right]^{1 / 2}\right\} d y d z .
$$

After symmetrization, the two points

$$
\left(x_{1}, y, z\right) \quad\left(x_{2}, y, z\right)
$$

of the original surface go into the points

$$
(-x, y, z) \quad(x, y, z)
$$

of the new, symmetrized surface where

$$
x=\left(x_{2}-x_{1}\right) / 2 .
$$

The area of the new surface is expressed by

$$
2 \iint\left[1+\left(\frac{\partial x}{\partial y}\right)^{2}+\left(\frac{\partial x}{\partial z}\right)^{2}\right]^{1 / 2} d y d z
$$

or, as we find using the abbreviations introduced, by

$$
\iint\left[4+\left(Y_{2}-Y_{1}\right)^{2}+\left(Z_{2}-Z_{1}\right)^{2}\right]^{1 / 2} d y d z .
$$

We assert that the integrand in (1) is greater than the integrand in (2). In fact, consider the two vectors with components

$$
1,-Y_{1},-Z_{1} \quad \text { and } \quad 1, Y_{2}, Z_{2} .
$$

Their resultant has the components

$$
2, Y_{2}-Y_{1}, Z_{2}-Z_{1} \text {. }
$$

Yet the sum of the lengths of two vectors can never be less than the length of their resultant. (The sum of two sides of a triangle never less than the third.) Therefore

$$
\left[1+Y_{1}^{2}+Z_{1}^{2}\right]^{1 / 2}+\left[1+Y_{2}^{2}+Z_{2}^{2}\right]^{1 / 2} \geq\left[4+\left(Y_{2}-Y_{1}\right)^{2}+\left(Z_{2}-Z_{1}\right)^{2}\right]^{1 / 2} .
$$

And so the integrand in the integral (1), expressing the surface-area before symmetrization, is never less than the integrand in the integral (2), expressing the surface-area after symmetrization. This justifies assertion IV. ${ }^{5}$

3. Applications. We wish to draw some consequences from Steiner's theorems recalled in the foregoing section. We use the same coordinate system as before. We take the plane of the drawing in Fig. 2 as our horizontal $x, y$-plane. That part of this plane which is surrounded by the closed line $A B C$ will be called "the domain $D$ ". We consider a function

$$
z=f(x, y)
$$

which is positive within the domain $D$, but vanishes along the boundary of $D$ (the closed line $A B C$ ). The surface with equation (3) and the horizontal $x, y$-plane include a solid, (a "hill" represented by contour lines in Fig. 2) of which $D$ is the base. We sym-

"The terms "decrease" and "increase" are used here in the wide sense. Strictly speaking, we should have said "never increases" instead of "decreases", etc. 
metrize our hill with respect to the $y, z$-plane which cuts the horizontal plane along the $y$-axis (the line $A^{*} C^{*}$ of Fig. 2). By this symmetrization our hill goes over into a symmetrical hill, of which the base, surrounded by the closed line $A^{*} B^{*} C^{*}$ will be called the domain $D^{*}$. Let

$$
z=f^{*}(x, y)
$$

be the equation of the surface that bounds the symmetrized solid (hill) from above. Therefore, $f^{*}(x, y)$ vanishes along the boundary of $D^{*}$ (the line $A^{*} B^{*} C^{*}$ in Fig. 2 which shows a few more contour lines of the surface (4)). In brief, symmetrization with respect to the $y, z$-plane transforms the points $A, B$ and $C$, the line $A B C$, the domain $D$ and the function $f(x, y)$ into the points $A^{*}, B^{*}$ and $C^{*}$, the line $A^{*} B^{*} C^{*}$, the domain $D^{*}$ and the function $f^{*}(x, y)$, respectively

Applying the results of the foregoing section to the present case of symmetrization, we observe that the volume remains unchanged (Sec. 2, III), or

$$
\iint_{\mathscr{D}} f d x d y=\iint_{D^{*}} f^{*} d x d y
$$

Yet the area is diminished (Sec. 2, IV), that is

$$
\iint_{\mathscr{D}}\left[1+f_{x}^{2}+f_{y}^{2}\right]^{1 / 2} d x d y \geq \iint_{D^{*}}\left[1+f_{x}^{*^{2}}+f_{y}^{*^{2}}\right]^{1 / 2} d x d y
$$

where $f_{x}=\partial f / \partial x, f_{y}, f_{x}^{*}$, and $f_{y}^{*}$ denote partial derivatives. Inequality (6) mentions only the curved part of the boundary of the solids compared; but the bases, the area of $D$ and that of $D^{*}$, are known to be equal (Sec. 2, I). This remark completes the derivation of (6).

Relation (5) will be useful later. We shall find another useful relation if we apply the inequality (6) not to $f$, but to $\epsilon f$, where $\epsilon$ is an arbitrary positive constant. We obtain

$$
\iint_{D^{2}}\left[1+\epsilon^{2}\left(f_{x}^{2}+f_{y}^{2}\right)\right]^{1 / 2} d x d y \geq \iint_{D^{*}}\left[1+\epsilon^{2}\left(f_{x}^{* 2}+f_{\nu}^{* 2}\right)\right]^{1 / 2} d x d y .
$$

We suppose that $\epsilon$ is sufficiently small, expand both sides of (7) in powers of $\epsilon$, observe that $D$ and $D^{*}$ have the same area (Sec. 2, I) and, after obvious cancellation and division by $\epsilon^{2}$, we obtain

$$
\frac{1}{2} \iint_{D}\left(f_{x}^{2}+f_{y}^{2}\right) d x d y+\cdots \geq \frac{1}{2} \iint_{D^{*}}\left(f_{x}^{*^{2}}+f_{y}^{*^{2}}\right) d x d y+\cdots .
$$

On both sides, subsequent terms, which are not written, only indicated, contain $\epsilon^{2}$ or higher powers of $\epsilon$. We let $\epsilon$ tend to 0 , and obtain finally

$$
\iint_{D}\left(f_{x}^{2}+f_{y}^{2}\right) d x d y \geq \iint_{D^{*}}\left(f_{x}^{*^{2}}+f_{\nu}^{*^{2}}\right) d x d y .
$$

This result will turn out to be extremely important in what follows.

By the way, instead of supposing $\epsilon$ small, we could have supposed it large. Doing this, we obtain, from (7)

$$
\iiint_{D}\left[f_{x}^{2}+f_{y}^{2}\right]^{1 / 2} d x d y \geq \iint_{D^{*}}\left[f_{x}^{*^{2}}+f_{\psi^{2}}^{*^{2}}\right]^{1 / 2} d x d y
$$


Dividing both sides by the area of $D$, or, what is the same, by the area of $D^{*}$, we can state the result by saying that symmetrization diminishes the average slope. This observation is quite instructive in showing the smoothing effect of symmetrization, but (5) and (9) will prove more important.

4. Parallel characterization of some physical quantities. Before we can derive the main result, we must make preparations of another kind. The aim of the present section is to characterize certain physical quantities so that their analogy, which is not obvious at first sight, should clearly appear.

I. We consider a homogeneous and isotropic elastic cylinder of which the crosssection is a plane domain $D$. When this cylinder is twisted around an axis perpendicular to $D$, the couple resisting the twist is $\theta_{\mu} \mathrm{P}$. In this formula $\theta$ denotes the twist, that is, the angle of rotation per unit length, $\mu$ the modulus of rigidity (or sheer), and $\mathrm{P}$ a purely geometric constant, depending on size and shape of the domain D. I propose to call $P$ the torsional rigidity of the cross-section $\mathfrak{D}$. The usual term for $\mu \mathrm{P}$ is torsional rigidity of the elastic cylinder. (There seems to be no generally accepted symbol for torsional rigidity. We use here $\mathrm{P}$, which is the Greek capital corresponding to the initial of "rigidity".)

In investigating the equilibrium position of the twisted cylinder, we can start from the general principle of minimum potential energy. Following the usual reasoning, but using a slightly different notation, we arrive at the following characterization of $P$, the torsional rigidity of the cross-section $D$.

Let $f(x, y)$ be an arbitrary function (just sufficiently "smooth" inside $D$ ) such that

$$
f(x, y)=0 \text { on the boundary of } \mathfrak{D} \text {. }
$$

Then

$$
\frac{\iint\left(f_{x}^{2}+f_{y}^{2}\right) d x d y}{4\left(\iint f d x d y\right)^{2}} \geq \frac{1}{\mathbf{P}}
$$

where both integrals are extended over the domain D. Equality in (11) is attained if, and only if, the function $f(x, y)$ is proportional to the stress-function, that is, if $f=$ const. $U$, where $U$ satisfies the differential equation

$$
U_{x x}+U_{y y}+2=0
$$

inside $D$ and satisfies the same boundary condition to which $f$ is subjected according to (10). (Of course, $U_{x x}=\partial^{2} U / \partial x^{2}$.) That is, $U$ must vanish along the boundary of $D$.

II. We consider a uniform and uniformly stretched elastic membrane, which is fixed along the curve surrounding $D$ and coincides with $D$ in its equilibrium position. Let $\Lambda$ denote the frequency of the principal tone of the membrane; the principal tone is the gravest proper tone of the membrane. In computing $\Lambda$, we drop a certain physical constant, that depends on the tension and density of the membrane and has the dimension of a velocity. Thus $\Lambda$ becomes a purely geometric quantity, depending on the shape and size of the domain $D$; it is characterized by the inequality

$$
\frac{\iint\left(f_{x}^{2}+f_{y}^{2}\right) d x d y}{\iint f^{2} d x d y} \geq \Lambda^{2}
$$

where both integrals are extended over the domain $D$. This inequality (13) holds for any function $f$ of the same nature as considered before; $f$ is arbitrary inside $D$ but 
satisfies the boundary condition (10). Equality in (13) is attained if, and only if, the function $f(x, y)$ is proportional to the transverse displacement in the principal vibration, that is, if $f=$ const. $v$, where $v$ is positive inside $D$, satisfies there the differential equation

$$
v_{x x}+v_{y y}+\Lambda^{2} v=0
$$

and vanishes along the boundary of $\mathfrak{D}$. Thus, $v$ is also subject to the boundary condition imposed upon $f$ by (10).

III. We consider finally two infinite cylinders, with parallel generatrices, one containing the other. We take the generatrices parallel to the $z$-axis. Then these cylinders intersect the $x, y$-plane in two closed curves, one contained in the other. Let us call $a$ the ring-shaped plane domain contained between these two curves. (In Fig. 2, we take the curve $A B C$ as outer boundary of $R$, and the innermost of the closed lines drawn inside $A B C$ as inner boundary.) We conceive the two cylinders as conductors of electricity, both having constant potential; the potential of the outer cylinder is 0 , that of the inner one different from 0 . The cylinders form a condenser and we call $C$ the capacity of this condenser per unit height.

Also $C$ can be characterized by an inequality. Let $f(x, y)$ be an arbitrary function (just smooth inside $R$ ) and such that

$f(x, y)=0$ along the outer,

$f(x, y)=f_{1}$ along the inner

boundary of $\mathcal{Q}$ where $f_{1}$ is a constant, different from 0 . Then

$$
\frac{\iint\left(f_{x}^{2}+f_{y}^{2}\right) d x d y}{4 \pi f_{1}^{2}} \geq C
$$

the integral is extended over the ring-shaped domain $R$. Inequality (15) holds for an arbitrary function $f(x, y)$ of the described type. Equality in (15) is attained if, and only if, $f(x, y)$ is proportional to the electrostatic potential, that is, if $f=$ const. $w$, where $w$ satisfies the differential equation

$$
w_{x x}+w_{y y}=0
$$

inside $R$ and boundary conditions of the same nature as $f(x, y)$ :

boundary of $R$.

$$
\begin{array}{ll}
w=0 & \text { on outer, } \\
w=1 & \text { on inner, }
\end{array}
$$

5. Influence of symmetrization on some physical quantities. After the preparations in the foregoing sections, we are now in a position to derive the following statements which are analogous to Steiner's Theorems discussed in Sec. 2.

I. Symmetrization increases the torsional rigidity.

II. Symmetrization decreases the principal frequency.

III. Symmetrization decreases the electrostatic capacity.

In brief, we assert that symmetrization decreases all three quantities, $1 / P, \Lambda$ and $C$, characterized by the parallel inequalities (11), (13) and (15) and connected with the corresponding differential equations (12), (14) and (16). The analogy so disclosed is an essential part of the proof. In fact, after having considered carefully the derivation of Theorem I, concerned with torsional rigidity, we shall need only a few additional remarks about II and III. 
We consider now two elastic shafts of which the cross-sections are ldisplayed in Fig. 2: the domain $D$, bounded by the curve $A B C$, and $D^{*}$, boumed $b y A^{*} B^{*} C^{*}$ Let $\mathrm{P}$ and $\mathrm{P}^{*}$ denote the torsional rigidities corresponding to $D$ and $D^{*} ;$ respectively.

As we have discussed in Sec. $4(\mathrm{I})$, there is a function $f(x, y)$, satisfying the boundary condition (10), and such that

$$
\frac{1}{\mathrm{P}}=\frac{\iint_{D}\left(f_{x}^{2}+f_{y}^{2}\right) d x d y}{4\left(\iint_{D} f d x d y\right)^{2}}
$$

This $f$ is the true stress-function corresponding to the crosis-section/D, or proportional to it. Thus, in Fig. 2, the contour lines inside $A B C$ represent the actual stress distribution in the twisted shaft with cross-section $D$.

Now, we symmetrize the domain $D$ and the function $f(x ; y)$ as deseribed in Sec. ' 3 ; and obtain $D^{*}$ and $f^{*}(x, y)$, respectively. The symmetrized domain $\mathscr{D}^{*}$ is ineluded by the line $A^{*} B^{*} C^{*}$ in Fig. 2. The symmetrized function $f^{*}(x ; y)$; is positive insidel $D^{*}$; and vanishes along its boundary. From the relations (5), and!(9), derived in 'Sec: 3, we wen-; clude that

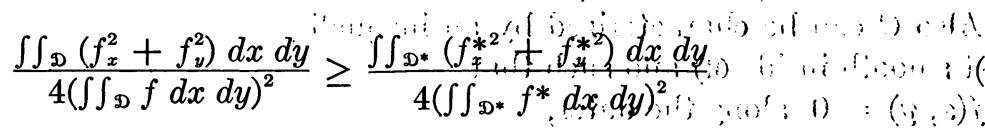

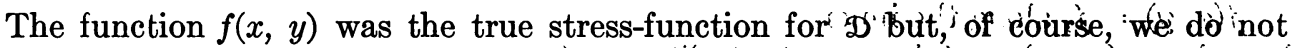
know whether $f^{*}$ is or is not the stress fun'tion' for $\mathscr{D}$ : *: (Very likedy it is nót. We have no reason to suppose that the contour lines inside $A^{*} B^{*} Q^{*}$, obtained by symmetrization, represent the true stress distribution.) Yet, at any rate, we can apply the inequality (11) characterizing the torsional rigidity to the case of the domain $D^{*}$, and so we obtain that

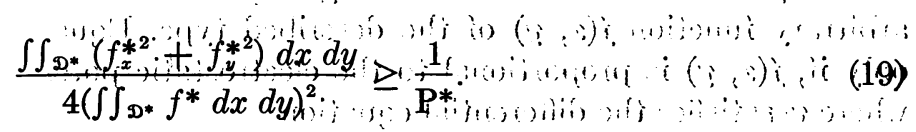

Joining (17), (18) and (19), we conclude that

$$
\text { , : , } \frac{1}{\mathrm{P}} \geq \frac{1}{\mathrm{P}^{*}}
$$

or

$$
\therefore: \mathbf{P}^{*} \geq \mathbf{P} \text {. }^{\prime}
$$

We have justified our assertion I.

Only small nodifications of the foregoing reasoning are needed to handle the cases of principal frequency and electrostatic eapacity, and to derive the corresponding Theorems II and III. To prove II, we must use, instead of (5), that

$$
\iint_{2} f^{2} d x d y=\iint_{D^{*}} f^{* 2} d x d y
$$

Yet this is evident. Indeed, when the surface with equation

is symmetrized the surface with equation

$$
z=[f(x, y)]^{2}
$$


is symmetrized along with it-a fact that we could express with the equation

$$
\left(f^{2}\right)^{*}=\left(f^{*}\right)^{2} .
$$

Therefore, (20) follows from the proposition that the volume is preserved by symmetrization (Sec. 2, III).

6. Applications. A few examples shall illustrate the general results of the foregoing section.

I. For a rectangle, both the torsional rigidity and the principal frequency have been found, but for a rhombus none of these two important quantities has been exactly expressed yet as far as I know. From Fig. 3, however, we can derive limits for them.

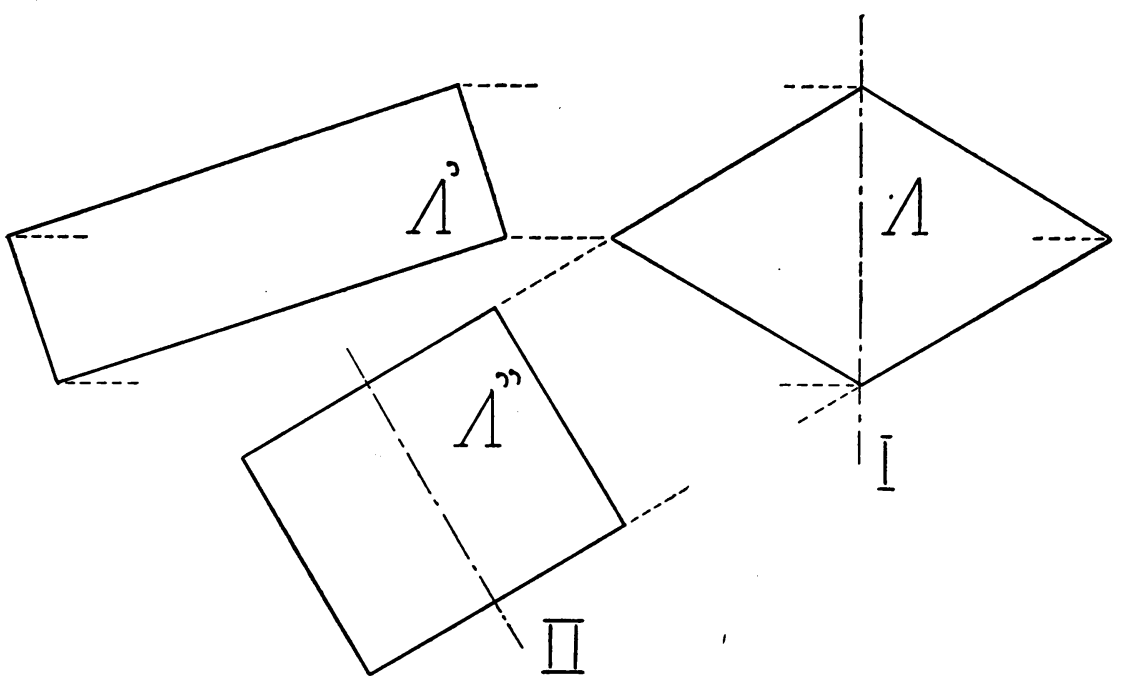

Fra. 3

Let $\Lambda, \Lambda^{\prime}$ and $\Lambda^{\prime \prime}$ denote the principal frequencies of the figures marked with these letters, respectively. Symmetrized with respect to the straight line I, the rectangle with principal frequency $\Lambda^{\prime}$ is transformed into a rhombus, and the latter, symmetrized with respect to II, into the other rectangle. Symmetrization, however, decreases the fundamental frequency (Sec. 5, II). Therefore

$$
\Lambda^{\prime}>\Lambda>\Lambda^{\prime \prime}
$$

Let $d$ denote the shorter diagonal and $\delta$ the acute angle of the rhombus. A little elementary geometry is enough to compute the sides of the two rectangles, after which (21) yields the following bounds for $\Lambda$, the principal frequency of the rhombus:

$$
\frac{2 \pi}{d}>\Lambda>\frac{\pi}{d} \frac{\left(1+\sin ^{2} \delta\right)^{1 / 2}}{\cos (\delta / 2)} .
$$

The lower bound is much nearer to the truth. In fact, it attains the exact value for $\Lambda$ in both extreme cases, for $\delta=\pi / 2$ (square) and for $\delta=0$ (infinite strip). The upper bound yields the exact value only for $\delta=\pi / 2$ (square).

[By other means, namely by conformal representation, as it will be explained in 
the joint paper quoted under ${ }^{2}$, we can derive an upper bound for $\Lambda$ which is much better than the bound $2 \pi / d$, except in cases in which $\delta$ is very near to $\pi / 2$ and the rhombus is almost a square:

$$
\Lambda<\frac{\pi}{d} \frac{j}{2 \pi^{1 / 2}} \Gamma\left(\frac{1}{2}-\frac{\delta}{2 \pi}\right) / \Gamma\left(1-\frac{\delta}{2 \pi}\right)
$$

$j=2.4048$ is the first positive zero of the Bessel function $J_{0}(x)$.

The following short table shows which degree of approximation we can obtain by combining the upper bound (23) with the lower bound of (22).

$\begin{array}{rcc}\delta & \frac{\left(1+\sin ^{2} \delta\right)^{1 / 2}}{\cos (\delta / 2)} & \frac{j \Gamma[(\pi-\delta) / 2 \pi]}{2 \pi^{1 / 2} \Gamma[(2 \pi-\delta) / 2 \pi]} \\ 90^{\circ} & 2.0000 & 2.0071 \\ 89 & 1.9826 & 1.9898 \\ 88 & 1.9654 & 1.9728 \\ 87 & 1.9483 & 1.9562 \\ 86 & 1.9313 & 1.9400 \\ 85 & 1.9145 & 1.9241 \\ 80 & 1.8322 & 1.8491 \\ 75 & 1.7525 & 1.7810 \\ 70 & 1.6752 & 1.7190 \\ 65 & 1.6002 & 1.6621 \\ 60 & 1.5273 & 1.6100 \\ 45 & 1.3256 & 1.4758 \\ 30 & 1.1575 & 1.3674 \\ 15 & 1.0419 & 1.2778 \\ 0 & 1.0000 & 1.2024\end{array}$

The first column expresses $\delta$ in degrees, the third in radians.

Taking the mean between the two bounds, we are certain to obtain the value of $\Lambda$ for a rhombus with an error of less than $1 \%$ as long as $\delta$ is not less than $75^{\circ}$.]

Also the torsional rigidity of a rhombic cross-section can be included between two limits by using Fig. 3.

II. Figure 4 shows how a quadrilateral can be transformed into a rhombus by two successive symmetrizations. Now, by Fig. 3, the rhombus so obtained can be symmetrized into a rectangle, the rectangle into another rhombus, this into another rectangle, and so on. As Fig. 3 suggests, the rectangles successively generated by this process become more and more square-like, and it is easy to prove that we obtain a square in the limit. Now, each of these successive symmetrizations preserves the area, increases the torsional rigidity and decreases the principal frequency. Therefore, our process shows: Of all quadrilaterals with a given area, the square has the maximum torsional rigidity and the minimum principal frequency.

A similar, but more obvious reasoning applies to triangles; compare, in Fig. 1, the general $\triangle A B C$ with the isosceles $\triangle A^{*} B^{*} C^{*}$. An appropriate process of successive symmetrization shows that of all triangles with a given area, the equilateral triangle has the highest torsional rigidity and the lowest principal frequency. Yet we could not handle in 
this manner the case of pentagons. Symmetrizing an irregular pentagon, we cannot avoid changing it into some polygon with more than five sides. (In Fig. 1, a pentagon is changed into an octagon.)

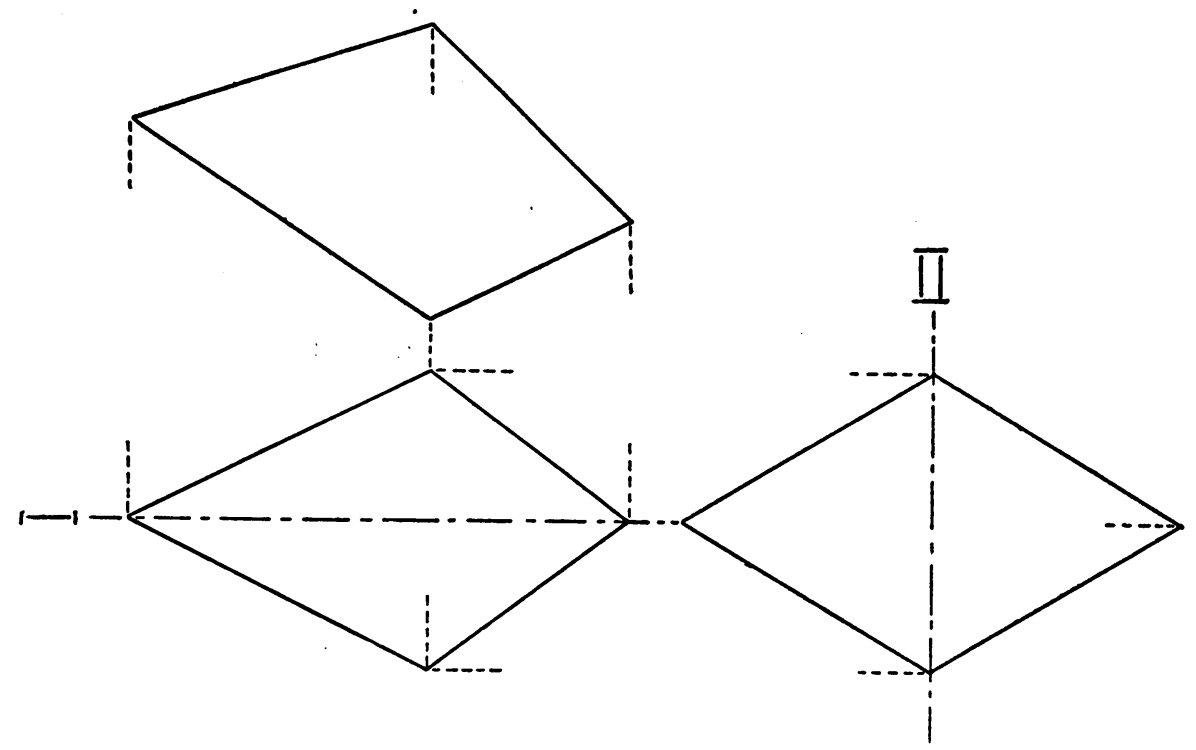

FIg. 4

It is natural to suspect that the propositions just proved about the equilateral triangle and the square can be extended to regular polygons with more than four sides. Yet the difficulty that we face when attempting the extension to pentagons can make us realize that our theorems on symmetrization are far from being trivial.

III. A circle is symmetric with respect to any of its diameters and remains unchanged by symmetrization with respect to any line whatsoever. On the other hand, by an appropriate (infinite) sequence of symmetrizations, any plane figure can be changed into a circle ultimately. (This is plausible, and has been proved.) Therefore, of all plane domains with a given area, the circle has the maximum torsional rigidity and the minimum principal frequency.

The second part of this statement, concerning principal frequency, has been conjectured by Lord Rayleigh, and later proved by Faber. The first part, concerning torsional rigidity, has been clearly foreseen by Saint-Venant who supported it by convincing physical considerations which, however, do not amount to a mathematical proof, and, to my knowledge, the first such proof is the one just presented. 